\title{
Prophylactic effects of thymoquinone against carbon tetrachloride- induced hepatic damage in Sprague-Dawley rats
}

\author{
Khaled M. A. Hassanein ${ }^{1 *}$, Ahmed Al-Emam², Khaled Radad ${ }^{1}$ \\ ${ }^{1}$ Pathology and Clinical Pathology Department, Faculty of Veterinary Medicine, Assiut University, Assiut 71526, Egypt. ${ }^{2}$ Department of Forensic Medicine \\ and Toxicology, Faculty of Medicine, Mansoura University, Mansoura, Egypt.
}

\begin{tabular}{l} 
ARTICLE INFO \\
\hline Article history: \\
Received on: $09 / 12 / 2015$ \\
Revised on: $27 / 12 / 2015$ \\
Accepted on: $12 / 01 / 2016$ \\
Available online: $27 / 02 / 2016$ \\
\hline Key words: \\
Carbon tetrachloride, \\
hepatotoxicity, histopathology, \\
thymoquinone, ultrastructure.
\end{tabular}

\section{INTRODUCTION}

Liver as a vital organ in the body playing a central role in metabolic homeostasis and detoxification of a variety of drugs and xenobiotics is vulnerable to a wide range of toxic, microbial, metabolic, circulatory and neoplastic insults (Taub, 2004; Shamsi-Baghbanan et al., 2014). Carbon tetrachloride $\left(\mathrm{CCl}_{4}\right)$, an industrial solvent, is a hepatotoxic agent and its administration is widely used as an animal model of toxin-induced liver injury that allows the evaluation of both necrosis and subsequent inflammation (Huh et al., 2004). $\mathrm{CCl}_{4}$-induced hepatic damage is widely used for hepatoprotective drug screening. Hepatotoxicity of $\mathrm{CCl}_{4}$ involves its biotransformation into free radicals such as trichloromethyl free radical $\left(\mathrm{CCl}_{3}\right)$ and trichloroperoxyl radical $\left(\mathrm{CCl}_{3} \mathrm{O}_{2}-\right)$, and increased lipid peroxidation (Feng et al., 2010). Herbal products have been used in traditional folk medicine for centuries to maintain health or to treat various human diseases

\footnotetext{
* Corresponding Author

Khaled M. A. Hassanein, Pathology and Clinical Pathology Department, Faculty of Veterinary Medicine, Assiut University, Assiut 71526, Egypt. Email: kh-hassanien@hotmail.com
}

(Myagmar et al., 2004). Currently, much attention has been paid to traditional herbal medicine for treating liver diseases. This is due to: (i) conventional and/or synthetic drugs can cause serious side effects especially when used for prolonged periods of time and (ii) discovery and development of modern technology which led to isolation and purification of many active ingredients from medicinal herbs (Sehrawat et al., 2006). TQ, the major active compound derived from the medicinal plant Nigella sativa, had anticancer effects against several cell lines and animal models (AbuKhader, 2013). The seeds of $N$. sativa have long been used in traditional medicine for a wide range of illnesses, including headache, dysentery, infections, obesity, back pain, bronchial asthma, hypertension and gastrointestinal problems (Al-Rowais, 2002).TQ was shown to protect against hepatic damage (Mansour et al., 2001). For instance, Ogus et al., (2012) demonstrated that oral administration of TQ relieved bile duct proliferation, periductular fibrosis and hepatic damage in bile duct-ligated rats. Lebda et al., (2011) reported that TQ prevented liver enzyme leakage and lipid peroxidation induced by D-galactosamine in rats. Nagi et al., (2010) observed that TQ protected mice against acetaminophen-induced hepatotoxicity. 
Helal (2010) attributed these effects of TQ to its antiinflammatory, anti-oxidant and anti-apoptotic properties. Nowadays, accidental hepatotoxicity that results from the exposure to toxic doses of hepatotoxins is significantly decreased due to increasing public awareness. On the other hand, mild hepatotoxicity results from some drugs, herbs or diets are popular and can chronically lead to apparent hepatic impairment. Accordingly, our current study tried to investigate the prophylactic effect of TQ against mild hepatic damage induced by oral administration of $\mathrm{CCl}_{4}$ in $\mathrm{SD}$ rats.

\section{MATERIALS AND METHODS}

All experimental procedures in the present study were done in accordance with the guidelines of the European Union Council (86/609/EU) and the manuscript was approved by the ethical committee (Assiut University, 7/2015). In which, 40 adult male SD rats $(180-230 g)$ were obtained from the Department of Pharmacology, Faculty of Medicine, Assiut University, Assiut, Egypt.

Rats were maintained under standard conditions with 12 h light/dark cycles and $22^{\circ} \mathrm{C}$, and $60 \%$ humidity. The food in the form of dry chow pellets and water were available at libitum. $\mathrm{CCl}_{4}$ $(\geq 99.9 \%)$ and TQ were obtained from Sigma-Aldrich (Germany).

\section{Study design and treatment protocol}

After acclimatization for one week, rats were randomly divided into 4 groups (10 rats each). The first group served as control and received daily normal saline $(0.5 \mathrm{ml} / \mathrm{rat})$ by gastric tube. The second group received $\mathrm{CCl}_{4}(2 \mathrm{ml} / \mathrm{kg}$ b.w.) twice a week on Sunday and Thursday by gastric tube (Singh et al., 2015). The third group was co-administered with $\mathrm{CCl}_{4}(2 \mathrm{ml} / \mathrm{kg}$ b.w. $)$ twice a week on Sunday and Thursday, and daily TQ $(20 \mathrm{mg} / \mathrm{kg} \mathrm{b.w.} \mathrm{in}$ corn oil) by gastric tube.

The fourth group received daily TQ $(20 \mathrm{mg} / \mathrm{kg} \mathrm{b.w.} \mathrm{in}$ corn oil) by gastric tube. After 5 weeks of treatment, rats were sacrificed, and blood samples and liver specimens were obtained for biochemical analysis and morphological examination, respectively.

\section{Measurement of serum ALT and AST activities}

Blood samples were allowed to clot and serum was obtained after centrifugation at $3000 \mathrm{rpm}$ for $15 \mathrm{~min}$. Serum ALT and AST activities were measured colorimetrically using ALT and AST assay kits according to the manufacturer (Sigma-Aldrich, Germany). Briefly, ALT and AST activity assays depend on the transfer of amino group from alanine and aspartate to $\alpha$-ketoglutarate resulting in generation of pyruvate and glutamate, respectively.

Generated pyruvate and glutamate are proportional to the activities of ALT and AST in the serum and can be colorimetrically measured using automatic biochemical analyzer (Hitachi 902, Roche Diagnostics, Germany) at wavelengths of 570 and $450 \mathrm{~nm}$ for ALT and AST, respectively.

\section{Histopathological examination}

Five different specimens were obtained from the liver of each rat. Specimens were fixed in $10 \%$ neutral buffered formalin for $24-48 \mathrm{~h}$, dehydrated in graded alcohol series, cleared in xylene and embedded in paraffin wax. Paraffinized specimens were cut at $4 \mu \mathrm{m}$ and tissue sections (one section/each specimen) were stained with hematoxylin and eosin (HE) (Bancroft and Stevens, 1990).

The stained sections were examined under light microscope (Olympus CX31, Japan) and photographed using digital camera (Olympus, Camedia-5060, Japan). The most common pathological findings were recorded.

\section{Transmission electron microscopy examination}

Also, five different specimens were obtained from the liver of each rat and immediately immersed in $2.5 \%$ gluteraldehyde solution for TEM. Specimens were then trimmed, fixed in gluteraldehyde solution in $0.1 \mathrm{M}$ sodium cacodylate buffer, $\mathrm{pH} 7.2$, and placed in a thermal box cooled to $4^{\circ} \mathrm{C}$ for $2 \mathrm{~h}$. They were post-fixed in $1 \%$ osmium tetraoxide in a sodium cacodylate buffer and then dehydrated in ascending series of ethyl alcohol and embedded in Spurr's resin.

Ultrathin sections stained with uranyl acetate and lead citrate were examined by TEM (JEOL100 CXII, Japan) operated at $80 \mathrm{KV}$ in the Electron Microscopy Unit, Pathology Department, College of Medicine, King Khalid University (Bancroft and Stevens, 1990). The most common ultrastructural findings were recorded as present or present.

\section{Statistical analysis}

Data were presented as means \pm standard error of means (SEM). When one-way ANOVA showed significant differences among groups, Duncan's post hoc test was used to determine the specific pairs of groups that were statistically different. Analysis was performed using statistical program SAS 1998. p<0.05 was considered as statistically signficant.

\section{RESULTS AND DISCUSSIONS}

\section{Serum ALT and AST activities}

Treatment of rats with $\mathrm{CCl}_{4}$ significantly increased serum ALT and AST activities by $14 \%$ and $19 \%$, respectively, compared to control rats (Fig. 1). On the other hand, concomitant treatment of rats with TQ and $\mathrm{CCl}_{4}$ significantly decreased serum ALT and AST activities by $12 \%$ and $14 \%$, respectively, compared to $\mathrm{CCl}_{4}$ treated rats (Fig. 1). TQ alone did not affect the activities of the serum enzymes (Fig. 1). In consistent, Cai et al., (2015) reported that elevation of serum ALT and AST activities is a constant finding following $\mathrm{CCl}_{4}$ treatment in different experimental animal models. ALT and AST are cytoplasmic aminotransferases that release extracellularly and go into the circulation upon hepatocytes damage. They are commonly used biomarkers for measuring hepatic injury in both experimental and clinical studies (Goorden et al., 2013). 


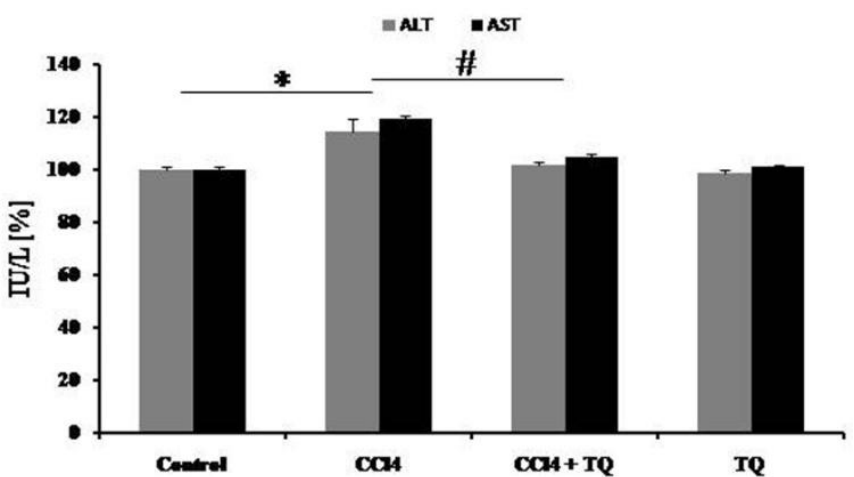

Fig. 1: Measurement of serum ALT and AST activities in treated rats. 100\% corresponds to the activities of ALT and AST in the serum of control rats. Values represent the mean \pm SEM of the serum ALT and AST activities in treated rats. $(* \mathrm{p}=0.01, \# \mathrm{p}=0.05)$

\section{Histopathology}

Light microscopic examination of HE-stained liver sections from control rats showed hepatic cells with a wellpreserved cytoplasm and well-defined nuclei (Fig. 2A). The most common histopathological findings as the result of $\mathrm{CCl}_{4}$ treatment were dilatation of blood sinusoids and portal blood vessels (Fig. 2B,C), activation of Kupffer cells (Fig. 2C), vacuolar degeneration of hepatocytes (Fig. 2D), focal areas of necrosis surrounded by a number of Kupffer cells (Fig. 3A) and mild portal fibrosis (Fig. 3B).
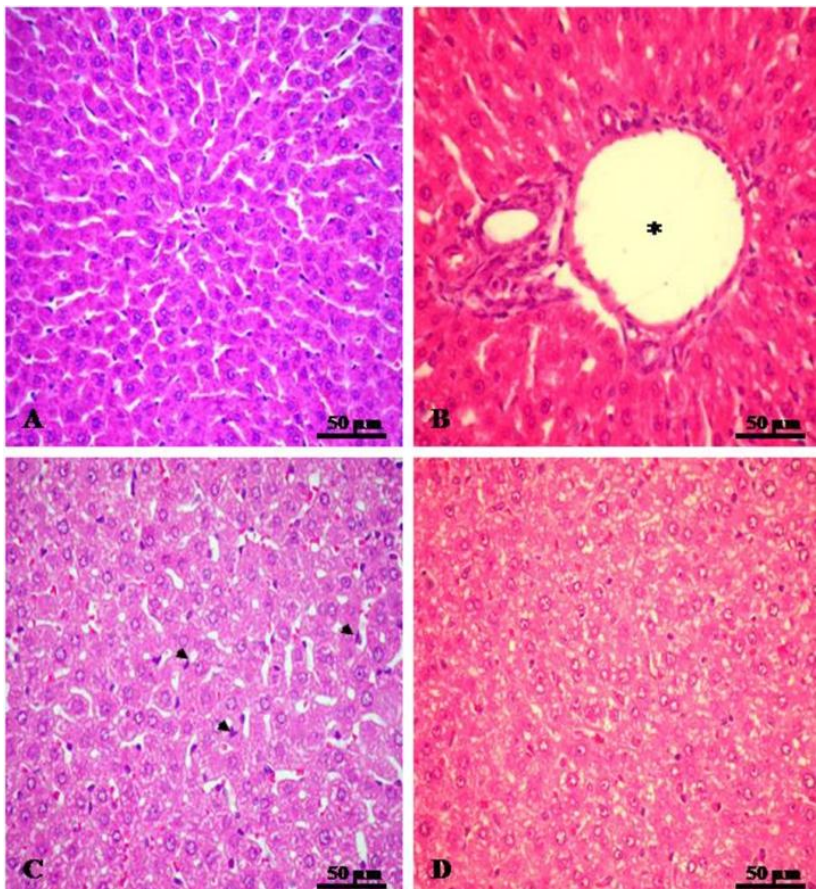

Fig. 2: Representative micrographs for histopathological changes in treated rats. A) Control rats showing normal hepatocytes with intact cytoplasm and centrally located vesicular nuclei. B) $\mathrm{CCl}_{4}$-treated rats showing dilatation of a portal vein (asterisk). C) $\mathrm{CCl}_{4}$-treated rats showing activation of Kupffer cells (arrow heads). D) $\mathrm{CCl}_{4}$-treated rats showing vacuolar degeneration of hepatocytes. HE.

Co-administration of rats with TQ and $\mathrm{CCl}_{4}$ attenuated $\mathrm{CCl}_{4}$-induced histopathological changes (Fig. 3C,D) and decreased their incidence compared to rats treated with $\mathrm{CCl}_{4}$ alone (Table 1). No apparent histopathological changes were seen in the liver of TQ-treated rats. In contrast to these mild hepatic changes, subcutaneous injection of $\mathrm{CCl}_{4}(2 \mathrm{ml} / \mathrm{kg}$ b.w. $)$ produced clear hepatic necrosis, inflammation, fatty accumulation and fibrosis after 12 weeks in rats (Tasci et al., 2008). Absence of severe histopathological changes including centrilobular necrosis and apparent fatty changes in our study was attributed to the oral route and short duration of $\mathrm{CCl}_{4}$ treatment.
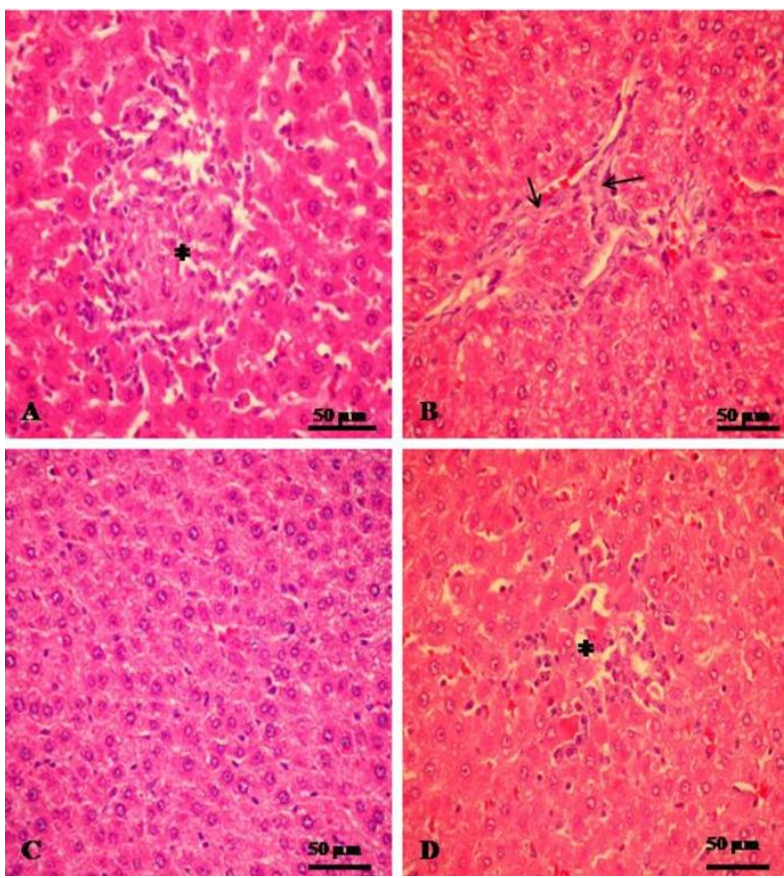

Fig. 3: Representative micrographs for histopathological changes in treated rats. A) $\mathrm{CCl}_{4}$-treated rats showing a focal area of necrosis surrounded by a number of Kuppfer cells (asterisk). B) $\mathrm{CCl}_{4}$-treated rats showing mild fibrosis in a portal area (arrows). C,D) Concomitant treatment of rats with TQ and $\mathrm{CCl}_{4}$ showing marked decrease of hepatic vacuolation and Kupffer cell activation, and attenuation of focal areas of necrosis (asterisk) HE.

Table 1: Incidence of main hepatic lesions in treated rats (5 tissue sections/ each treated rat).

\begin{tabular}{|c|c|c|c|c|}
\hline Lesions & Figures & 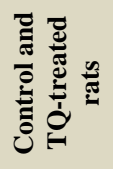 & 窇 & 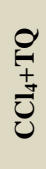 \\
\hline \multicolumn{5}{|l|}{ Light microscopy: } \\
\hline Portal vascular dilatation & Fig. 2B & - & +++ & + \\
\hline Kupffer cell activation & Fig. 2C & - & +++ & + \\
\hline Hepatic vacuolation & Fig.2D & - & +++ & + \\
\hline Focal areas of necrosis & Fig. $3 \mathrm{~A}$ & - & +++ & ++ \\
\hline Hepatic fibrosis & Fig. 3B & - & +++ & ++ \\
\hline \multicolumn{5}{|l|}{ Electron microscopy: } \\
\hline Dilatation of endoplasmic & Fig. 4B & - & +++ & + \\
\hline 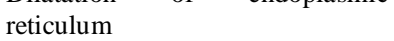 & Fig. $4 \mathrm{C}$ & - & +++ & + \\
\hline Extracellular matrix & Fig.4D & - & +++ & + \\
\hline $\begin{array}{l}\text { Fatty globules } \\
\text { Autophagosomes }\end{array}$ & Fig.4E & - & +++ & + \\
\hline
\end{tabular}

\begin{tabular}{ll}
\hline- & No lesions found in examined tissue sections \\
+ & Lesions found clearly in $5-15$ tissue sections $/ 10$ treated rats \\
++ & Lesions found clearly in $16-25$ tissue sections $/ 10$ treated rats \\
+++ & Lesions found clearly in $35-50$ tissue sections/10 treated rats \\
\hline
\end{tabular}




\section{Electron microscopy}

Normal hepatocytes appeared with intact cell membrane and many spherical mitochondria (Fig. 4A). The most common ultrastructural changes as the result of $\mathrm{CCl}_{4}$ treatment were in the form of dilatation of endoplasmic reticula (Fig. 4B), increased extracellular matrix (Fig. 4C), and formation of numerous perinuclear fatty globules (Fig. 4D) and autophagosomes (Fig. 4E). Co-administration of TQ and $\mathrm{CCl}_{4}$ relieved most of these changes and decreased their incidence compared to rats treated with $\mathrm{CCl}_{4}$ alone (Table 1). In which, TQ relieved dilatation of endoplasmic reticula and deposition of extracellular matrix (Fig. 4F).

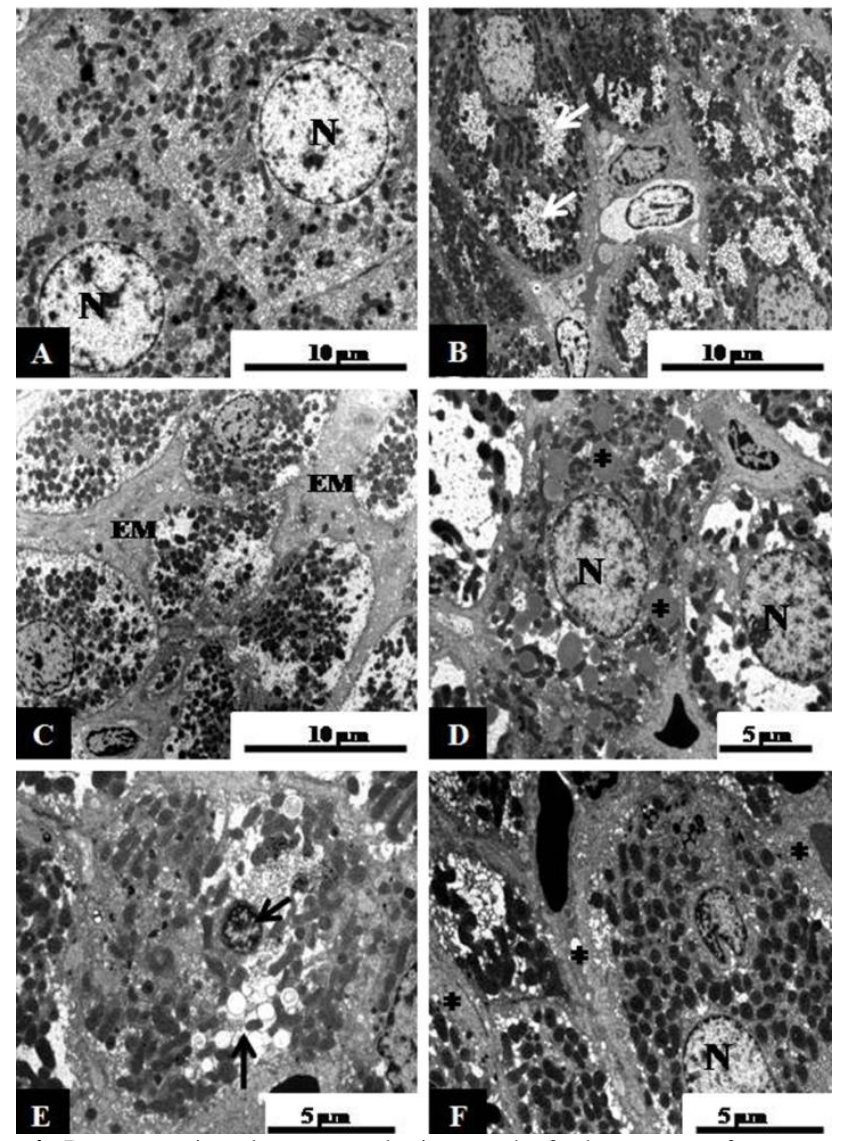

Fig. 4: Representative ultrastructural micrographs for hepatocytes from control and $\mathrm{CCl}_{4}$-treated rats. A) Normal hepatocytes showing intact cell membrane, centrally located nucleus $(\mathrm{N})$ and many spherical mitochondria. B) Hepatocytes from $\mathrm{CCl}_{4}$-treated rats showing dilatation of endoplasmic reticula (asterisks). C) Hepatocytes from $\mathrm{CCl}_{4}$-treated rats showing increase extracellular matrix (EM). D) A hepatocyte showing presence of numerous perinuclear fatty globules (asterisks). E) A hepatocyte showing presence of numerous autophagosomes (small arrows). F) Concomitant treatment of rats with TQ and $\mathrm{CCl}_{4}$ showing milder ultrastructural changes compared to rats treated with $\mathrm{CCl}_{4}$ alone. See mild dilatation of endoplasmic reticula and decreasing deposition of extracellular matrix (asterisks).

TQ-treated rats had no apparent changes by TEM. In consistent, Hsu (1998) and Knockaert et al., (2012) reported that vesicular changes of the endoplasmic reticulum and large lipid droplets were prominent ultrastructural changes as the result of $\mathrm{CCl}_{4}$ treatment in mice and rats, respectively. Increasing number of autophagosomes in the livers from $\mathrm{CCl}_{4}$-treated rats seemed to result from sequestration of damaged intracellular organelles.

\section{Effect of TQ against $\mathrm{CCl}_{4}$-induced liver damage}

Co-administration of TQ with $\mathrm{CCl}_{4}$ significantly lowered the activities of ALT and AST, and decreased the incidence of histopathological and ultrastructural changes compared to $\mathrm{CCl}_{4}$ treated rats. In consistent, Mansour et al., (2001) and El-Sayed (2011) reported that TQ significantly reduced $\mathrm{CCl}_{4}$-induced ALT and AST elevation in mice and rats, respectively. Essawy et al., (2012) found that aqueous extract of Nigella sativa ameliorated most histopathological changes induced by $\mathrm{CCl}_{4}$ in mice. This cytoprotective effect of TQ against $\mathrm{CCl}_{4}$-induced hepatic damage was reported to be attributed to the antioxidant effects of TQ. In this context, El-Sayed (2011) reported that TQ increased the activities and mRNAs levels of some antioxidant enzymes such as glutathione S-transferase, NAD (P) H-quinoneoxidoreductase and microsomal epoxide hydrolase. Al-Ghamdi (2003) found that TQ produced potent antioxidant effect against $\mathrm{CCl}_{4}$-induced free radical species.

In conclusion, the present study indicates that TQ has the potential to attenuate $\mathrm{CCl}_{4}$-induced hepatic damage in SD rats. Moreover, the results support the role of TQ as a nutritional supplement to prevent liver maladies.

\section{COMPETING INTERESTS}

The authors declared no potential conflicts of interest.

\section{REFERENCES}

Taub R. Liver regeneration: from myth to mechanism. Nat Rev Mol Cell Biol, 2004; 5: 836-847.

Shamsi-Baghbanan H, Sharifian A, Esmaeili S, Minaei B. Hepatoprotective herbs, avicenna viewpoint. Iran Red Crescent Med J, 2014; 16: e12313.

Huh CG, Factor VM, Sanchez A, Uchida K, Conner EA, Thorgeirsson SS. Hepatocyte growth factor/cmet signaling pathway is required for efficient liver regeneration and repair. Proc Natl Acad Sci USA, 2004; 101: 4477-4482.

Feng Y, Siu KY, Ye X, Wang N, Yuen MF, Leung CH, Tong Y, Kobayashi S. Hepatoprotective effects of berberine on carbon tetrachloride-induced acute hepatotoxicity in rats. Chin Med, 2010; 18: 33.

Myagmar BE, Shinno E, Ichiba T, Aniya Y. Antioxidant activity of medicinal herb rhodococcum vitis-idaea on galactosamineinduced liver injury in rats Phytomedicine, 2004; 11: 416-423.

Sehrawat A, Sharma S, Sultana S. Preventive effect of tannic acid on 2-acetylaminofluorene induced antioxidant level. tumor promotion and hepatotoxicity: a chemopreventive study. Redox Rep, 2006; 11: 85-95.

AbuKhader MM. Thymoquinone in the clinical treatment of cancer: Fact or fiction? Pharmacogn Rev, 2013; 7(14): 117-120.

Al-Rowais NA. Herbal medicine in the treatment of diabetes mellitus. Saudi Med J, 2002; 23: 1327-1331.

Mansour MA, Ginawi OT, El-Hadiyah T, El-Khatib AS, AlShabanah OA, Al-Sawaf HA. Effects of volatile oil constituents of Nigella sativa on carbon tetrachloride-induced hepatotoxicity in mice: evidence for antioxidant effects of thymoquinone. Res Commun Mol Pathol Pharmacol, 2001; 110: 239-251.

Ogus S, Kanter M, Erboga M, Erenoglu C. Protective effects of thymoquinone against cholestatic oxidative stress and hepatic damage after biliary obstruction in rats, J Mol Histol, 2012; 43: 151-159.

Lebda FM, Ahmed MA, Abd El Samad AA, Shawky MK. Protective effect of thymoquinone against D-galactosamine-induced liver injury in rats. Australian Journal of Basic and Applied Sciences, 2011; 5: 49-58. 
Nagi M., Almakki HA, Sayed-Ahmed MM, Al-Bekairi AM. Thymoquinone supplementation reverses acetaminophen-induced oxidative stress, nitric oxide production and energy decline in mice liver. Food Chemical Toxicology, 2010; 48: 2361-2365.

Helal GK. Thymoquinone supplementation ameliorates acute endotoxemia-induced liver dysfunction in rats. Pak J Pharm Sci, 2010; 23: 131-137.

Singh N, Khullar N, Kakkar V, Khaur IP. Sesamol loaded solid lipid nanoparticles: a promising intervention for control of carbon tetrachloride induced hepatotoxicity. BMC Complement Altern Med, 2015; 3: 142 .

Bancroft JD, Stevens A. Theory and Practice of Histological Technique. Edinburg: Churchil Livingstone, 1990: 113-305.

Cai Z, Lou Q, Wang F, Li E, Fang H, Xi J, Ju L. Nacetylcysteine protects against liver injure induced by carbon tetrachloride via activation of the Nrf2/HO-1 pathway. Int J Clin Exp Pathol, 2015; 8: 8655-8662.

Goorden SM, Buffart TE, Bakker A, Buijs MM. Liver disorders in adults: ALT and AST. Ned Tijdschr Geneeskd,. 2013; 157: A6443.

Tasci I, Mas N, Mas MR, Tuncer M, Comert B. Ultrastructural changes in hepatocytes after taurine treatment in $\mathrm{CCl}_{4}$ induced liver injury. World J Gastroenterol, 2008; 21: 4897-4902.

Hsu CT. Ultrastructural changes in liver damage induced by carbon tetrachloride in spontaneously hypertensive rats and Wistar-Kyoto rats. J Auton Nerv Syst, 1998; 28: 79-83.
Knockaert L, Berson A, Ribault C, Prost PE, Fautrel A, Pajaud J. Carbon tetrachloride-mediated lipid peroxidation induces early mitochondrial alterations in mouse. liver Lab Invest, 2012; 92: 396-410.

El-Sayed WM. Upregulation of chemoprotective enzymes and glutathione by Nigella sativa (black seed) and thymoquinone in $\mathrm{CCl}_{4-}$ intoxicated rats. Int J Toxicol, 2011; 30: 707-714.

Essawy AE, Abdel-Moneim AM, Khayyat LI, Elzergy AA. Nigella sativa seeds protect against hepatotoxicity and dyslipidemia induced by carbon tetrachloride in mice. J Applied Pharmaceutical Science, 2012; 2: 21-25.

Al-Ghamdi M. Protective effect of Nigella sativa seeds against carbon tetrachloride-induced liver damage. The American Journal of Chinese Medicine, 2003; 31: 721-728.

\section{How to cite this article:}

Hassanein KMA, Al-Emam A, Radad K. Prophylactic effects of thymoquinone against carbon tetrachloride-induced hepatic damage in Sprague-Dawley rats. J App Pharm Sci, 2016; 6 (02): 167-171. 